CERN-PPE/94-62

April 10, 1994

\title{
Test Beam Results for an Upgraded Forward Tagger of the L3 Experiment at LEP II
}

\author{
M. Chemarin ${ }^{1}$, P. Depasse ${ }^{1}$, J. Fay ${ }^{1}$, M. Felcini ${ }^{2 *}$, \\ L. Fredj ${ }^{3}$, B. Ille ${ }^{1}$, F. Nessi-Tedaldi ${ }^{4}$ and G. F. Susinno ${ }^{3}$ \\ ${ }^{1}$ IPN Lyon, IN2P3-CNRS, Université Claude Bernard, Lyon, France \\ ${ }^{2}$ CERN, PPE Division, Geneva, Switzerland \\ ${ }^{3}$ University of Geneva, Geneva, Switzerland \\ ${ }^{4}$ Eidgenössische Technische Hochschule, Zürich, Switzerland
}

\begin{abstract}
We have tested new scintillator modules with silicon photodiode readout for the upgraded Active Lead Rings (ALR) of the L3 detector at LEP II. Results are presented from data recorded in muon and electron test beams with particular emphasis on the light production and collection as a function of the particle impact position on the scintillator modules. The results from the beam test data will be used for the design of the readout and trigger electronics in conjunction with the required ALR performance as an electron tagger and beam background monitor at LEP II.
\end{abstract}

Submitted to Nuclear Instruments and Methods

* Now at Eidgenössische Technische Hochschule, Zürich, Switzerland 


\section{Introduction}

Silicon PIN photodiodes can be found in large quantities in several particle physics detectors. Their use is essentially limited to the read out of heavy inorganic scintillators as, for example, the $12000 \mathrm{BGO}$ crystals of the L3 electromagnetic calorimeters [1].

However it was soon established that this same readout technique can also be used successfully for plastic scintillators [2]. This is very useful in situations where severe space limitations and the existence of a magnetic field exclude the use of bulky devices like photomultiplier tubes. This is the case in the L3 experiment, where sandwiches of lead and scintillators are directly read out through PIN photodiodes. These sensors are insensitive to magnetic fields and, due to the absence of any gain, very stable and very reliable.

The detectors hereafter called Active Lead Rings (ALR) cover the forward and backward region between $4^{\circ}$ and $9^{\circ}$, as described elsewhere [3]. Originally the Active Lead Rings were installed to protect the inner tracking chamber from stray background particles. Instrumenting the lead material with plastic scintillators allowed us to use them as vetoes, improving on the hermiticity of the whole L3 detector. The elements consist of 3 scintillator layers $10 \mathrm{~mm}$ thick placed behind $18.5 \mathrm{~mm}\left(3.3 X_{0}\right)$ thick lead converters. The scintillators, which have trapezoidal shape, are read out at their outer edge by photodiodes of dimension $10 \times 20 \mathrm{~mm}^{2}$. The present detector has only $\phi$ segmentation with layers of the type shown on the left of Fig. 1.
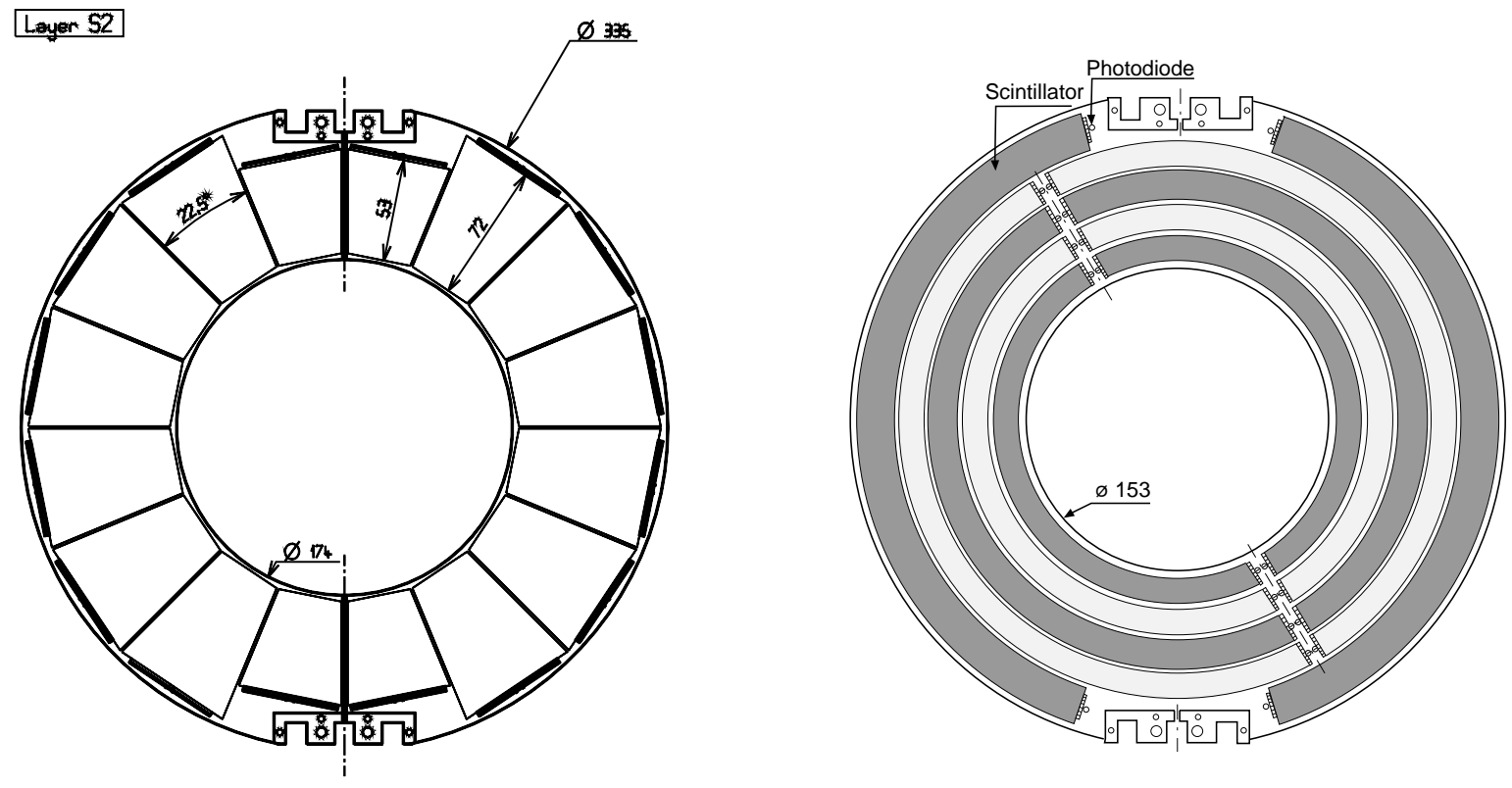

Figure 1: $x-y$ views of the scintillator layers for a module of the upgraded ALR. Shown on the left is a layer segmented in $\phi$ while a layer segmented in $\theta$ is shown on the right.

An upgrade has been proposed mainly for two physics motivations [4]: 
- to improve hermiticity for new particle searches,

- to extend the $Q^{2}$ range for the measurement of the real photon structure function.

The latter requires a segmentation in polar angle $\theta$ of the ALR scintillators. We have proposed to use the same technique and to construct semicircular elements with photodiode readout at both ends to obtain $\theta$ information. A view of an ALR scintillator layer with $\theta$ segmentation is shown on the right of Fig. 1.

Before choosing the final configuration, the light yield and the uniformity of the response with the distance to the photodiode need to be known. In particular, it is important [4] to be able to observe minimum ionizing particles.

For this purpose, tests with muons (at $225 \mathrm{GeV}$ ) and electrons (at 20 and $40 \mathrm{GeV}$ ) were performed in late 1993. The results are presented below.

\section{Experimental setup}

\subsection{The beam line}

The beam tests were performed in the H2 North Hall beam line of the CERN SPS. Beam particles are tagged by a set of five scintillators (S1-S5) (see Fig. 2). A triple coincidence $\mathrm{S} 1-\mathrm{S} 2-\mathrm{S} 3$ was used to define a wide beam $\left(2 \times 2 \mathrm{~cm}^{2}\right.$ wide strips $)$ while the addition of S4 and $\mathrm{S} 5\left(2 \times 0.5 \mathrm{~cm}^{2}\right.$ wide strips $)$ in cross allowed to have a narrow beam $\left(5 \times 5 \mathrm{~mm}^{2}\right)$.

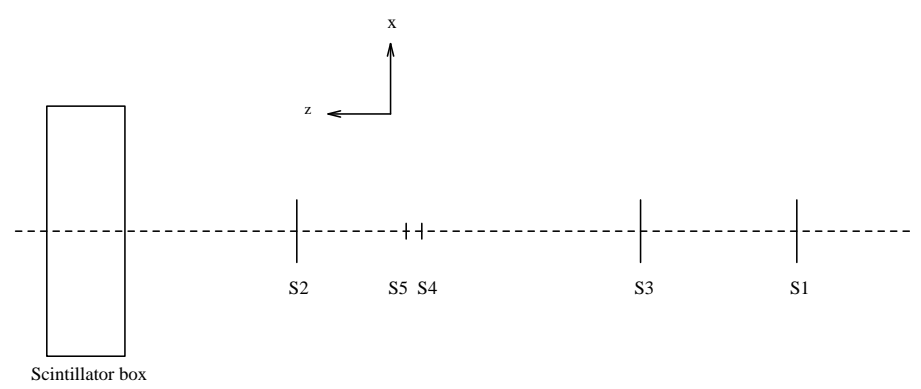

Figure 2: Beam line set-up.

\subsection{The ALR scintillators}

A box containing the prototype scintillators was placed, at a distance of $60 \mathrm{~cm}$ downstream from S5, on a horizontally and vertically movable table allowing us to vary the beam impact point on the ALR scintillators. Each motor step corresponds to $1.05 \mathrm{~mm}$, providing a precise knowledge of the relative displacement of the apparatus with respect to the beam. 

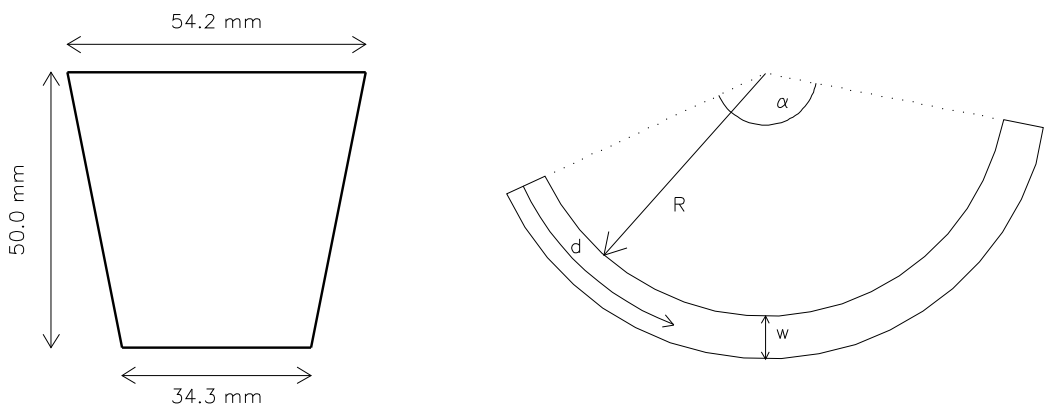

Figure 3: Dimensions of the "Phi" module and definition of the parameters for the semicircular scintillators.

\begin{tabular}{|c|c|c|c|c|c|c|}
\hline Scintillator & $\begin{array}{c}\text { Inner } \\
\text { radius } \\
(\mathrm{mm})\end{array}$ & $\begin{array}{c}\text { Width w } \\
(\mathrm{mm})\end{array}$ & $\begin{array}{c}\text { angle } \\
\alpha\end{array}$ & Wrapping & $\begin{array}{c}\text { ADC } \\
\text { channel } \\
\text { number }\end{array}$ & $\begin{array}{c}\text { Photodiode } \\
\text { sensitive } \\
\text { area }\end{array}$ \\
\hline \hline $\mathrm{A}$ & 83 & 16 & $180^{\circ}$ & $\begin{array}{c}\text { Aluminized } \\
\text { mylar }\end{array}$ & $\begin{array}{c}1 \\
6\end{array}$ & $20 \times 7.5 \mathrm{~mm}^{2}$ \\
\hline $\mathrm{B}$ & 83 & 16 & $180^{\circ}$ & $\begin{array}{c}\text { Aluminized } \\
\text { mylar }\end{array}$ & 2 & $70 \times 10 \mathrm{~mm}^{2}$ \\
\hline $\mathrm{C}$ & 149 & 26 & $180^{\circ}$ & Millipore [8] & 3 & $20 \times 7.5 \mathrm{~mm}^{2}$ \\
\hline $\mathrm{D}$ & 148 & 26 & $144^{\circ}$ & $\begin{array}{c}\text { Aluminized } \\
\text { mylar }\end{array}$ & $\begin{array}{c}4 \\
9\end{array}$ & $20 \times 7.5 \mathrm{~mm}^{2}$ \\
\hline $\mathrm{E}$ & 120 & 26 & $180^{\circ}$ & $\begin{array}{c}\text { Aluminized } \\
\text { mylar }\end{array}$ & 5 & $20 \times 7.5 \mathrm{~mm}^{2}$ \\
\hline $\mathrm{F}$ & - & - & - & Millipore [8] & 8 & $20 \times 7.5 \mathrm{~mm}^{2}$ \\
\hline
\end{tabular}

Table 1: Dimensions and characteristics of the tested scintillator modules. See Fig.3 for the definition of $\mathrm{R}, \mathrm{w}$ and $\alpha$. 
In a light-tight box, five different semicircular scintillator prototypes were tested together with a "Phi" one, similar to those already present in the existing ALR (see Fig. 3 for dimensions). The dimensions and characteristics of the 5 semicircular scintillator modules (labelled from A to E) and of the "Phi" module (labelled F) are given in Table 1. As scintillator material, NE102 [5] was used for A,B,C,D and E and BC408 [6] was used for F, all $1 \mathrm{~cm}$ thick.

These scintillators were read out with silicon photodiodes [7]; A, B, D and E had 2 photodiodes, one at each end, while only one end of scintillator $\mathrm{C}$ was equipped with a photodiode.

Two different wrapping options were tested. In one case, the scintillator was covered with an aluminized mylar layer to insure good light reflection from the scintillator surface. In the other case, the scintillator was wrapped in millipore [8], as are the scintillators for the existing ALR.

\section{$2.3 \quad$ Electronics}

The electronic chain associated with the photodiodes consisted of charge preamplifiers [9] and of standard RC-CR shaping amplifiers [10]. The time constant of the shapers was chosen to be around $2 \mu \mathrm{s}$, well optimized to detector capacities ranging from $50 \mathrm{pF}$ up to $200 \mathrm{pF}$. The charge preamplifier boards were located just behind the scintillators, inside the box, at a distance not exceeding $10 \mathrm{~cm}$. The shapers, 3 meters away, gave 2 output signals: one positive energy signal with variable gain (from 1 up to 1600) and one "fast" negative signal for self-triggering purposes, useful for the calibration of each chain with gamma sources or cosmic rays. The digitization was performed by $2 \mathrm{CAMAC}$ peak sensing ADCs (11 bits ORTEC ADC811), located $\sim 70 \mathrm{~m}$ away in the counting room. The data acquisition was performed by a VME system [11], based on a 68040 microprocessor with an OS9 (Version 2.4) operating system. The data were written to EXABYTE cassettes for off-line analysis. A diagram of the read out electronics is shown in Fig. 4.

\section{Calibration procedure.}

A ${ }^{57}$ Co source was placed on the photodiode of scintillator F. ${ }^{57} \mathrm{Co}$ is a $122 \mathrm{keV} \gamma$ source (it emits also $14 \mathrm{keV}$ and $137 \mathrm{keV} \gamma$ 's but with smaller probability). Since $3.6 \mathrm{eV}$ are needed to produce a p-n pair in silicium, the ${ }^{57}$ Co source produces a charge of $\sim 33500$ electrons in the photodiode. An example of a spectrum obtained in a calibration run with the ${ }^{57} \mathrm{Co}$ source is shown in Fig. 5. A high precision pulser was used to produce a signal of peak pulse height equal to the one given by the gamma source. This signal was then attenuated by given factors in order to obtain a calibration curve.

The pulser signal was injected into the calibration line of the charge preamplifiers (PAC). The calibration line setup is schematically shown in Fig. 4. During calibration, the photodiode input to the PAC was connected, thus the width of the signal received gives a good estimate of the electronic noise. When a minimum ionizing particle (MIP) 


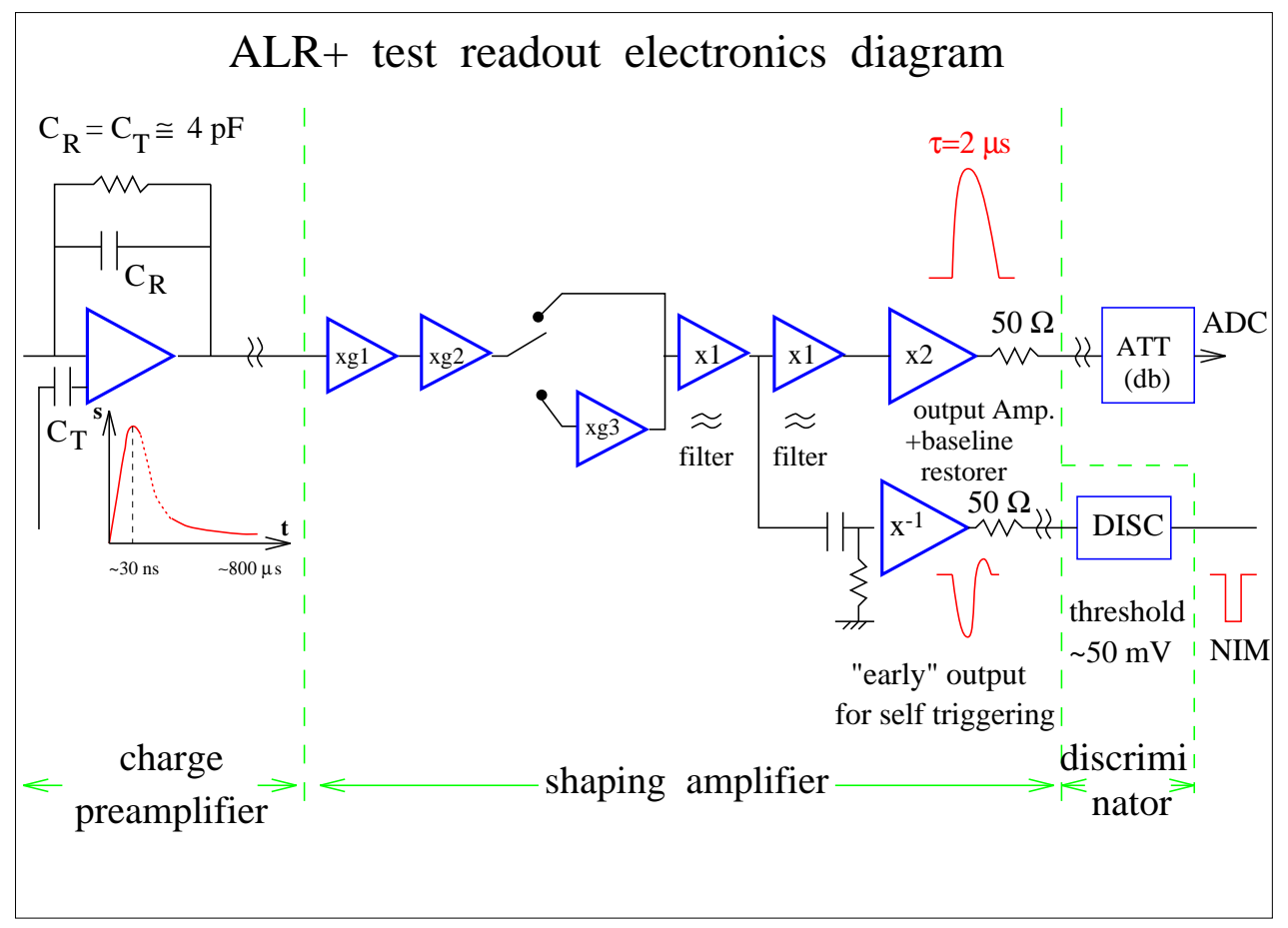

Figure 4: Diagram of the read out electronics.

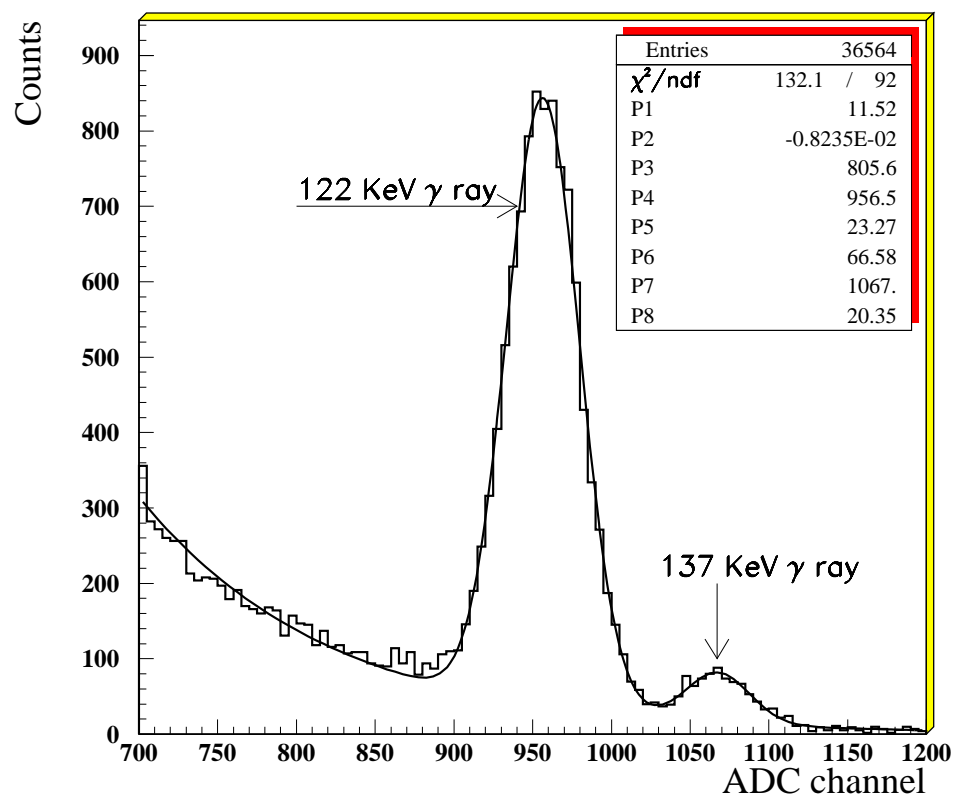

Figure 5: Spectrum obtained in a calibration run with the ${ }^{57} \mathrm{Co}$ source. 

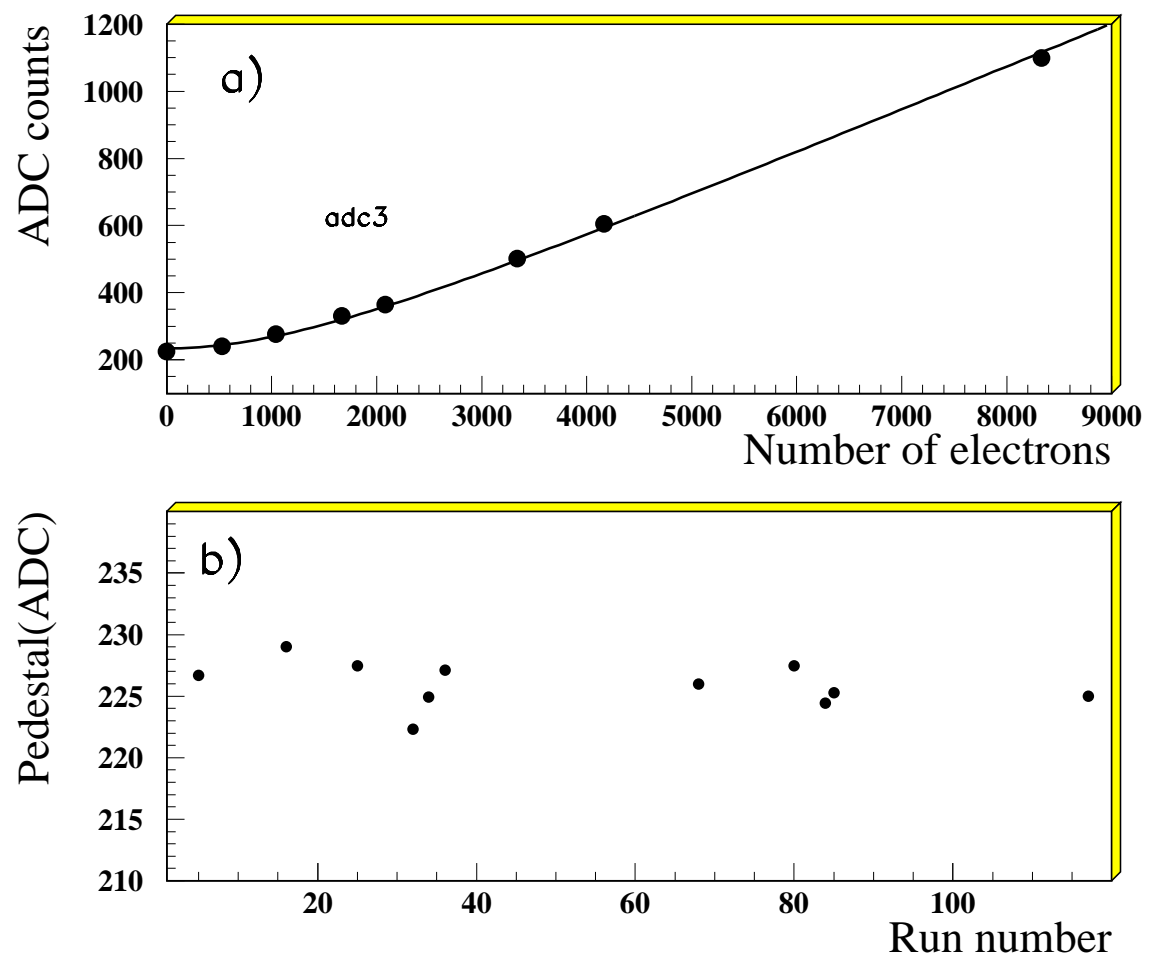

Figure 6: a) Calibration curve and b) pedestal stability for scintillator $\mathrm{C}$

\begin{tabular}{|c|c|c|}
\hline $\begin{array}{c}\text { ADC channel } \\
\text { Number }\end{array}$ & $\begin{array}{c}\mathrm{a} \\
\text { (electrons) }\end{array}$ & $\mathrm{b}$ \\
\hline \hline 1 & 620 & 208 \\
\hline 2 & 700 & 155 \\
\hline 3 & 550 & 239 \\
\hline 4 & 660 & 159 \\
\hline 5 & 690 & 167 \\
\hline 6 & 630 & 167 \\
\hline 7 & 830 & 143 \\
\hline 8 & 690 & 164 \\
\hline 9 & 610 & 202 \\
\hline 10 & 650 & 163 \\
\hline
\end{tabular}

Table 2: Fitted calibration parameters. 


\begin{tabular}{|c|c|c|}
\hline Scintillator & ADC & Noise (electrons) \\
\hline \hline $\mathrm{A}$ & 1 & $771 \pm 5$ \\
& 6 & $823 \pm 6$ \\
\hline $\mathrm{B}$ & 2 & $724 \pm 5$ \\
& 7 & $718 \pm 5$ \\
\hline $\mathrm{C}$ & 3 & $744 \pm 5$ \\
\hline $\mathrm{D}$ & 4 & $777 \pm 5$ \\
& 10 & $799 \pm 6$ \\
\hline $\mathrm{E}$ & 5 & $738 \pm 5$ \\
& 9 & $812 \pm 6$ \\
\hline $\mathrm{F}$ & 8 & $819 \pm 6$ \\
\hline
\end{tabular}

Table 3: Noise estimated from runs with $1 / 4$ attenuation factor.

crosses a scintillator module, the signal produced ( $\sim 2000$ electrons per MIP) lies in the transition region between the linear and the non linear output regime of the readout system (mainly due to the baseline restorer of the shaper amplifiers), as can be seen in Fig. 6-a).

The calibration data were fitted with a hyperbole, where two parameters define the calibration curve:

$$
N_{e}=a \cdot \sqrt{\frac{A D C^{2}}{b^{2}}-1},
$$

where $N_{e}$ is the number of electrons at the PAC input, $a$ and $b$ are the fitted parameters and ADC is the ADC channel number. The fitted function is superimposed to the measured points in Fig. 6-a). The fitted parameters for the various ADC channels are listed in Table 2.

The width of a pulse height spectrum is a measure of the electronic noise for the experimental setup. The estimated noise for each channel is reported in Table 3.

Since for a millipore wrapped scintillator, a MIP produces a signal with a typical magnitude of 2300 electrons (or 1800 electrons for an aluminized mylar wrapped scintillator), we can deduce that the signal to noise ratio $(\mathrm{S} / \mathrm{N}$ ) is about 3 (2 for aluminized mylar wrapping).

Pedestal runs were taken approximately three times per day to monitor the pedestal stability. Pedestals were found to be stable within an interval of \pm 5 ADC counts, as shown in Fig. 6-b). This fluctuation is negligible compared to the electronic noise of 66 ADC counts. 


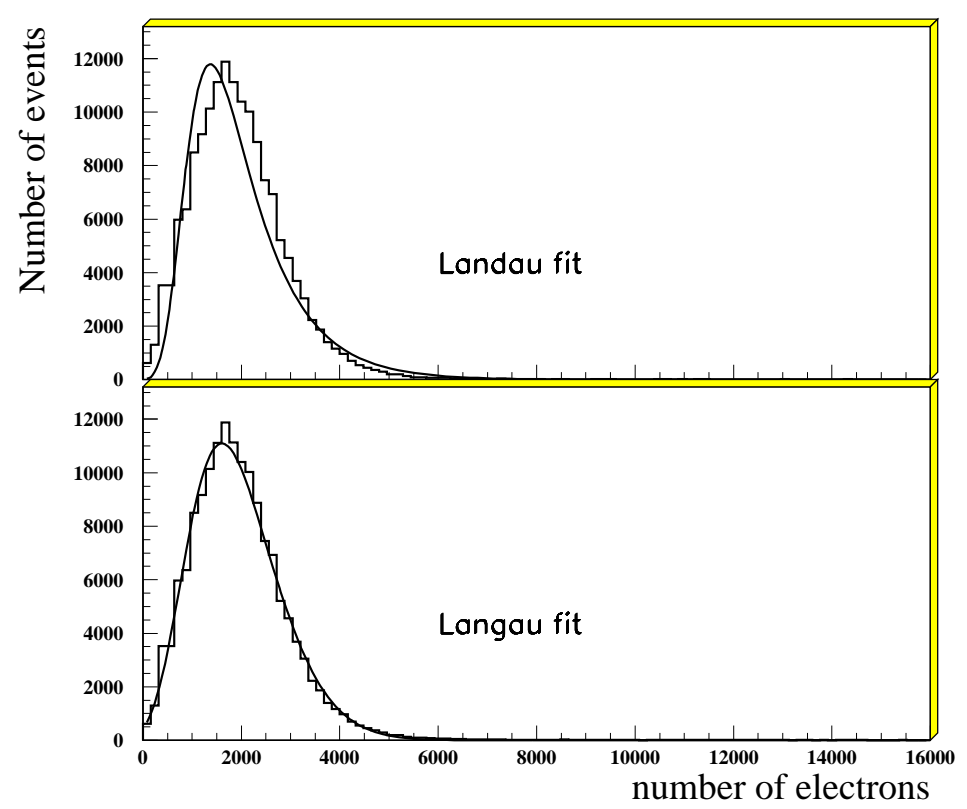

Figure 7: Typical muon spectrum. A Landau fit is overlayed in the upper plot while a langau fit is overlayed in the lower plot.

\section{Tests with muons}

The light yield was determined for the various scintillators which differ in radius, width, angle $\alpha$ and wrapping, as shown in Table 1. Amplification factors in the electronic chain were $\mathrm{g} 1=2, \mathrm{~g} 2=18$ and $\mathrm{g} 3=22$ (see Fig. 4 ).

\subsection{Fitting method}

The calibration distributions were fitted using a function, hereafter called langau, which is a product of a standard Landau with a gaussian distribution centered on the Landau's most probable value. The noise contamination of the physical distribution is thus taken into account. The formula for the langau function is:

$$
F(x)=\underbrace{A \cdot e^{\left(\frac{-x+e(-x)}{2}\right)}}_{\text {LANDAU }} \cdot \underbrace{e^{\left(-\frac{x^{2}}{2 \sigma^{2}}\right)}}_{\text {GAUSSIAN }},
$$

where $x=\mathrm{R} \cdot\left(N_{e}-\overline{N_{e}}\right)$, with $\overline{N_{e}}$ the most probable value, $\mathrm{R}$ a fitting constant and $\sigma$ the standard deviation of the gaussian distribution. As shown in Fig. 7, this method allows an improved estimate of the Landau parameters. The light yield values reported in the following correspond to the most probable values of the fitted langau function. 


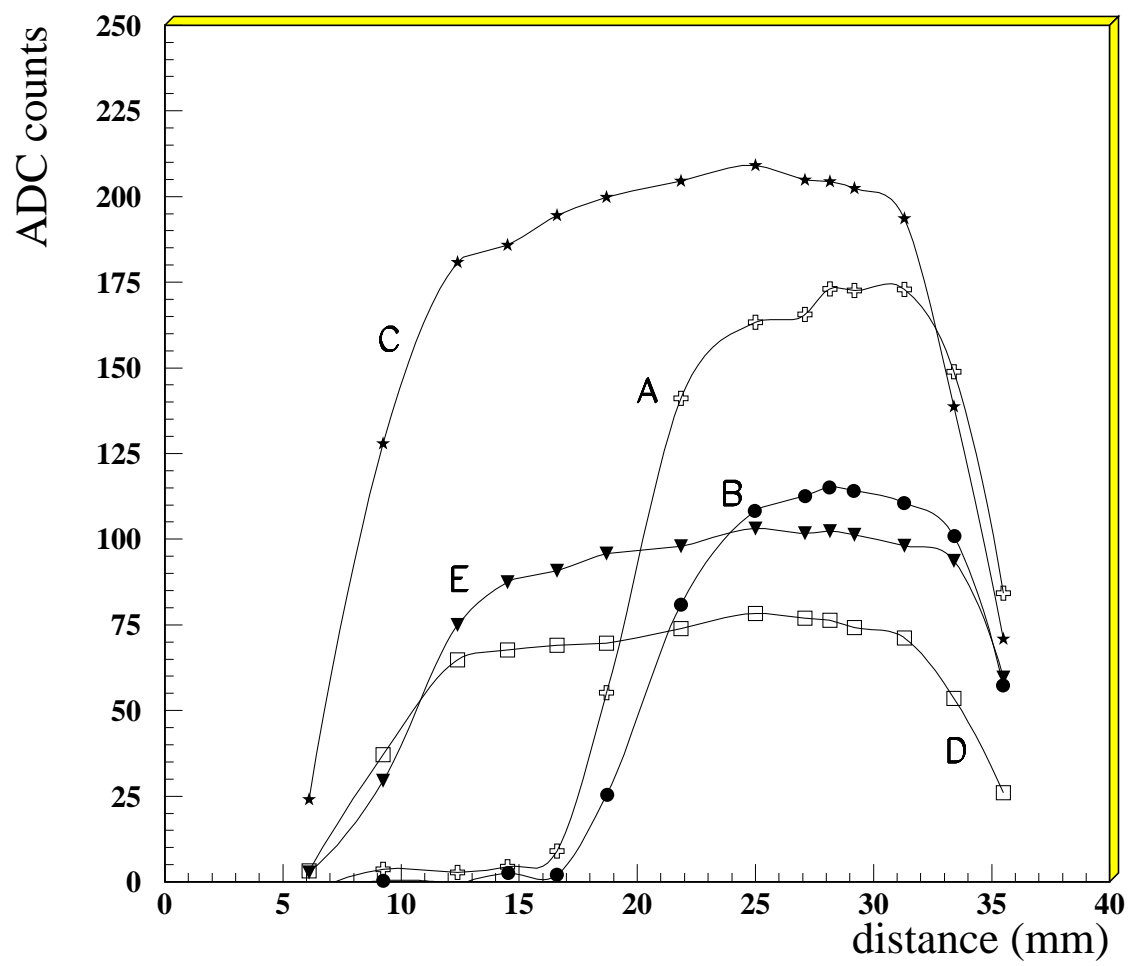

Figure 8: Results of a transverse scan (along the width w, see Fig. 3) with muon beam around point $\mathbf{p} 2$ with muons for the various $\theta$ scintillators (values are pedestal subtracted). Here small distance values correspond to the inner edge of the scintillator.

\section{2 $\theta$ module scan across its width}

All $\theta$ scintillators were scanned in width to obtain the maximum information about the position dependence of the scintillator response. As a general feature, it was found that the light collection increases moving from the inner edge outwards. This effect is enhanced as the beam impact point is moved away from the photodiode, mainly because there is no direct light path from the impact point to the photodiode, and only photons selected by their maximum scattering angle reach the photodiode. This effect is, however, only of the order of few percent of the total light yield, as shown in Fig.8, where the results of a vertical scan across scintillator point p2 (see Fig.9) are shown.

\section{3 $\theta$ module scan along its length}

The dependence of the semicircular scintillator response on the impact point distance from the photodiode was analyzed. The positions of the various impact points on scintillator $\mathrm{C}$ are illustrated on Fig. 9. A parameter $\lambda$ is defined and is computed in the same way as the commonly used attenuation length, although we cannot truly speak in terms of attenuation length, which only applies to linear propagation in a given medium. So, if $d$ is the distance between the beam impact point and the photodiode along the semicircular scintillator (see Fig. 3), $N_{\epsilon}(d)$ the corresponding number of produced electrons in the photodiode and $\lambda$ the characteristic parameter, the following function can be fitted to the measured distribution: 

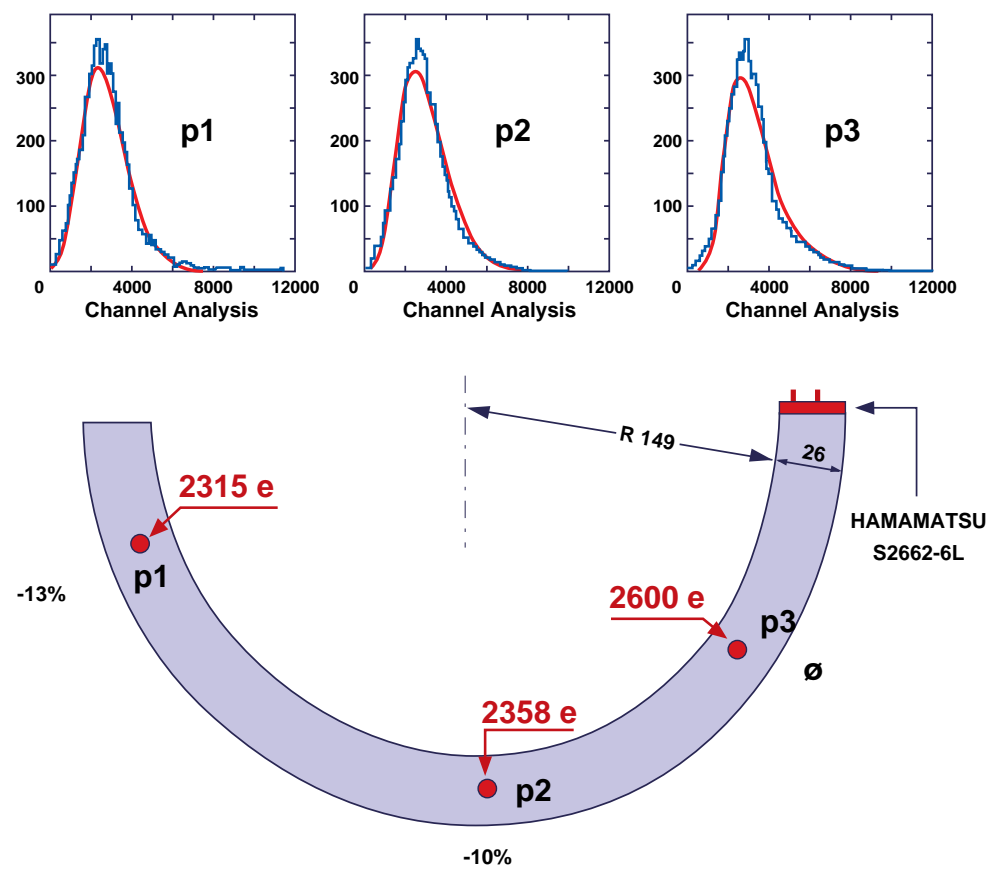

Figure 9: Results of a scan along the semicircle for scintillator C. Corresponding langau distributions from muons are shown on top.

$$
N_{e}(d)=A \cdot e^{\left(-\frac{d}{\lambda}\right)}
$$

The computed $\lambda$ parameters for all the semicircular scintillators are given in Table 4. Comparing Table 4 and Table 1 , it is evident that the most important contribution to the light uniformity is given by the wrapping. In fact the $\lambda$ value corresponding to the millipore wrapped scintillator $\mathrm{C}$ is at least four times larger than for any of the others. The uncertainty on the experimental setup metrology does not allow us to deduce, from the values in Table 4, a clear shape dependency.

The best results were obtained for scintillator $\mathrm{C}$, using a millipore wrapping. This scintillator setup gave a relative loss of light, from one end to the other, of the order of $13 \%$, while for the scintillator D the loss was about 26\%, as shown in Fig.10.

\subsection{Light yield of scintillator $\mathrm{F}$}

The first scintillator on the beam path was the scintillator $\mathrm{F}$, with a shape similar to those already used in the present Active Lead Rings.

The observed signal for scintillator $\mathrm{F}$, at the peak of the langau distribution, is $\sim 6000$ electrons, i.e. $3000 \mathrm{e} / \mathrm{MeV}$ (taking $2 \mathrm{MeV}$ as the energy loss of muons in $1 \mathrm{~cm}$ of scintillator). Very little variation was observed in a vertical scan. The measured light yield for the "Phi" scintillator modules of the present ALR is $\sim 4000 \pm 180 \mathrm{e} / \mathrm{MeV}$ (average over 25 modules of the same dimensions). The factor 1.3 by which the observed 

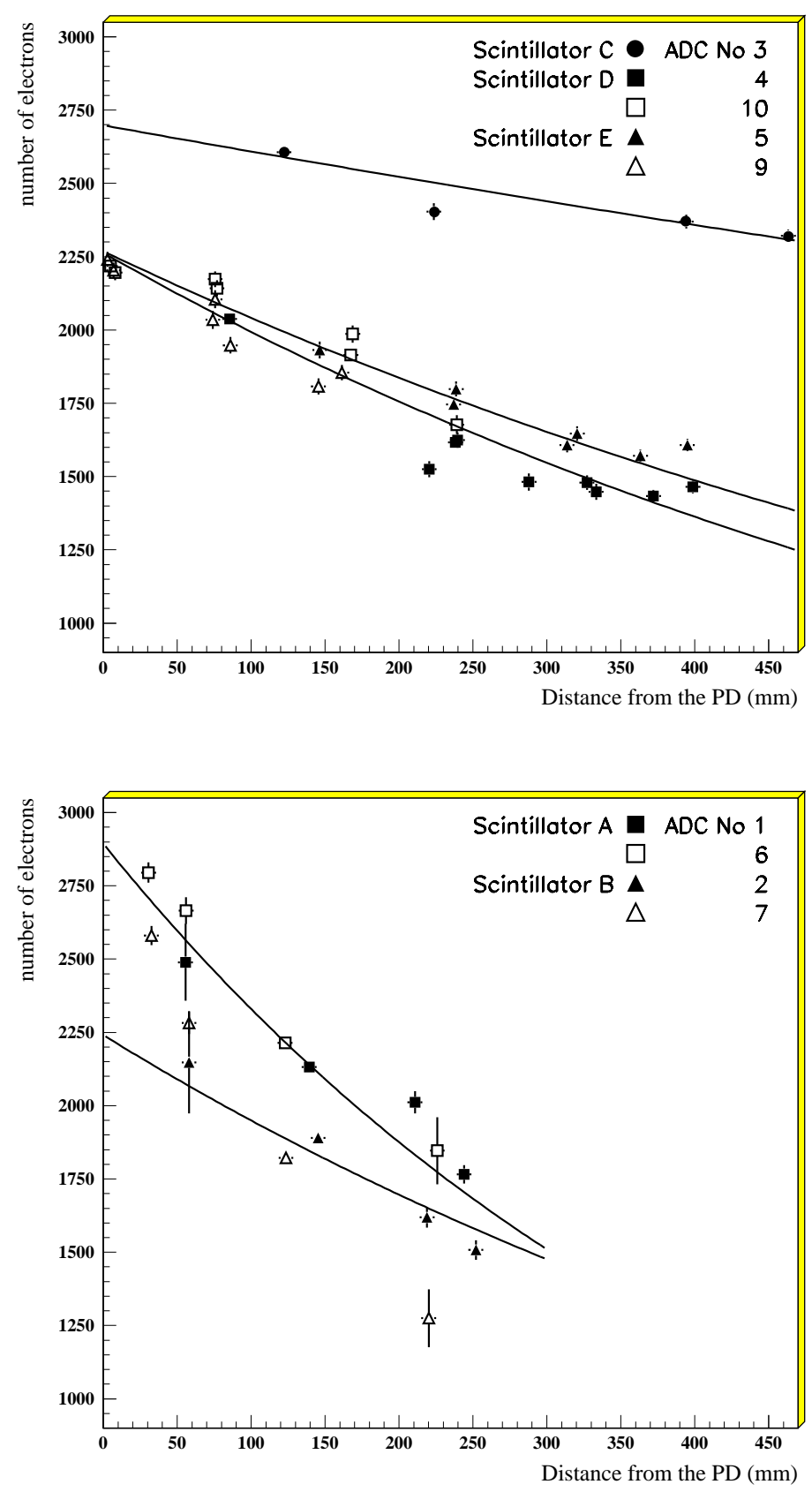

Figure 10: Observed number of electrons as function of the muon beam impact point distance from the photodiode, for the five semicircular scintillators: C, D, E (top) and A, B (bottom). 


\begin{tabular}{|c|c|}
\hline Scintillator & $\lambda(\mathrm{mm})$ \\
\hline \hline $\mathrm{A}$ & $460 \pm 20$ \\
\hline $\mathrm{B}$ & $720 \pm 40$ \\
\hline $\mathrm{C}$ & $2970 \pm 250$ \\
\hline $\mathrm{D}$ & $790 \pm 10$ \\
\hline $\mathrm{E}$ & $950 \pm 20$ \\
\hline
\end{tabular}

Table 4: The computed $\lambda$ parameters for all the semicircular scintillators. For scintillators A, B, D and E, equipped with 2 photodiodes (one at each end), the given value is the average between the two $\lambda$ values computed for the 2 ends.

light yield of scintillator $\mathrm{F}$ is reduced compared to the existing ALR modules can be explained by the fact that the scintillator $\mathrm{F}$ was read out by a single photodiode $\left(1.5 \mathrm{~cm}^{2}\right.$ active area) as compared to the 2 photodiode readout in the ALR setup.

\section{$5 \quad$ Tests with electrons}

The 6 plastic scintillator elements (A, B, C, D, E and F), placed behind a lead sheet of $24 \mathrm{~mm}\left(4.3 X_{o}\right)$ thickness, were exposed to electron beams of 20 and $40 \mathrm{GeV}$. The impact point was p2 (see Fig. 9). As the signal for electrons is much larger than in the muon case, the amplification path used for muons could no longer be used. Instead, the channel with $\mathrm{g} 1=2, \mathrm{~g} 2=18$ and $\mathrm{g} 3=1$ (see Fig. 4) was used. A dedicated ${ }^{57} \mathrm{Co}$ run and test pulse generator runs were performed to determine the gain of each channel in this configuration, using the method described in Section 3.

Both the electron beams of 20 and $40 \mathrm{GeV}$ were contaminated by hadrons. From the number of events in the minimum ionizing peak we estimate the percentage of non electron events to be $22 \%$ at $20 \mathrm{GeV}$ and $3.4 \%$ at $40 \mathrm{GeV}$.

The energy deposited in each scintillator, in $\mathrm{MeV}$, can be extracted for each channel $i(i=1$ to 10$)$ using the formula:

$$
E_{i}=\frac{g_{i}\left(n_{i}-p_{i}\right)}{c_{i}}
$$

where $n_{i}, g_{i}, p_{i}$ and $c_{i}$ are respectively the number of ADC counts, the conversion gain (in electrons/ADC channel), the pedestal and the calibration constant (in electrons/MeV) at point p2. The constants $p_{i}$ and $g_{i}$ were deduced from the dedicated ${ }^{57} \mathrm{Co}$ and test generator runs, and the $c_{i}$ values were obtained from the calibration runs with muons. The $c_{i}$ values are listed in Table 5. Fig. 11 shows the correlation between the 2 signals coming from scintillator $\mathrm{A}$ at $20 \mathrm{GeV}$.

In the case where the scintillator is read out at both ends (A, B, D and E) the same energy should be measured by the left and right channels. This is checked with the 


\begin{tabular}{|c|c|c|c|c|}
\hline \hline Scintillator & $\begin{array}{c}\text { channel } \\
\text { (left) }\end{array}$ & $\begin{array}{c}c_{i} \\
(\mathrm{e} / \mathrm{MeV})\end{array}$ & $\begin{array}{c}\text { channel } \\
\text { (right) }\end{array}$ & $\begin{array}{c}c_{i} \\
(\mathrm{e} / \mathrm{MeV})\end{array}$ \\
\hline \hline $\mathrm{A}$ & 1 & 1066. & 6 & 1107. \\
$\mathrm{~B}$ & 2 & 945. & 7 & 910. \\
$\mathrm{C}$ & 3 & 1185. & & \\
$\mathrm{D}$ & 4 & 808. & 10 & 942. \\
$\mathrm{E}$ & 5 & 873. & 9 & 957. \\
$\mathrm{~F}$ & 8 & 3043. & & \\
\hline \hline
\end{tabular}

Table 5: The calibration constants used in point $\mathbf{p} 2$ for the 10 channels.

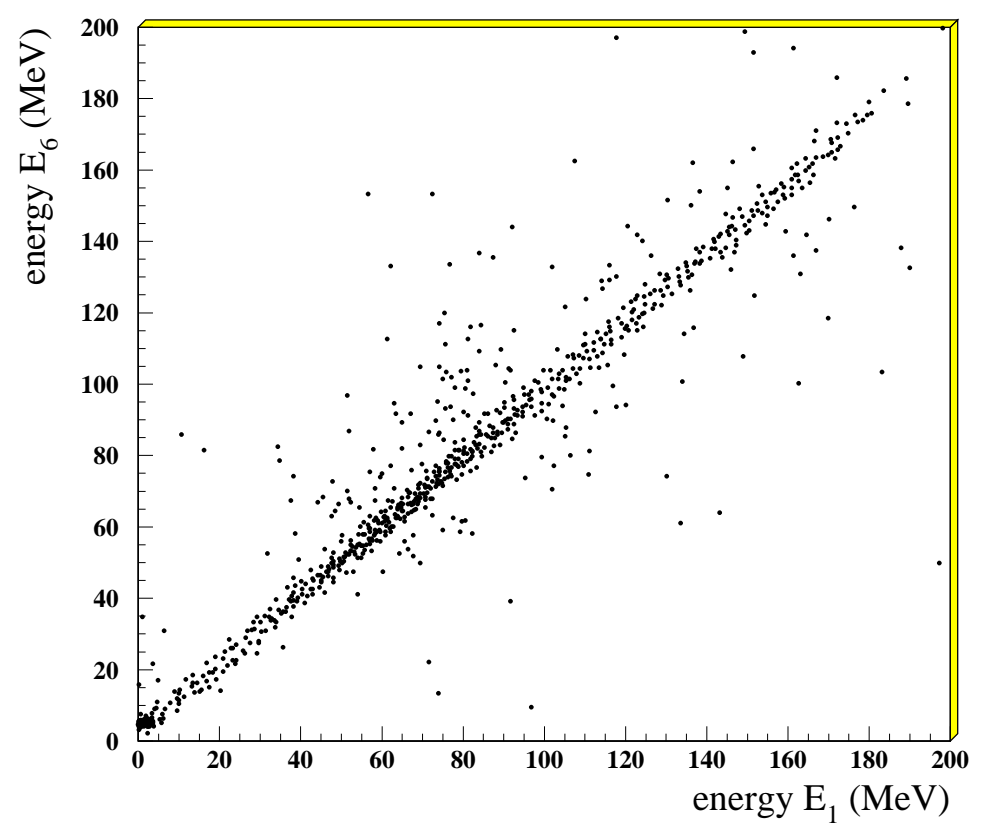

Figure 11: Correlation between the energies seen by the 2 channels looking at scintillator A for a $20 \mathrm{GeV}$ electron beam. 
asymmetry function

$$
y_{j}=\frac{\left(E_{L j}-E_{R j}\right)}{\left(E_{L j}+E_{R j}\right)}
$$

with $j=1,2,3,4$ for scintillators A,B,D and E respectively. Here $E_{L j}\left(E_{R j}\right)$ is the signal in the left (right) photodiode. The $y_{j}$ distribution must be centered at zero if the calibration is correct. The energy deposited in each scintillator is then defined as:

$$
E_{j}=\frac{\left(E_{L j}+E_{R j}\right)}{2}
$$

Fig. 12 shows the $y_{j}$ distributions for scintillators $\mathrm{A}, \mathrm{B}, \mathrm{D}$ and $\mathrm{E}$ at $40 \mathrm{GeV}$. The peak RMS is around 1.5\% and shifts away from zero of few \% are observed, showing imperfect calibration using muons. In fact having a data sample of minimum ionizing particles and electrons, an in-situ calibration can be performed constraining the energy $E_{j}$ in Eq. 3 to be $2 \mathrm{MeV}$ for one minimum ionizing particle and $y_{j}$ to be zero for events with electromagnetic shower energy deposition.
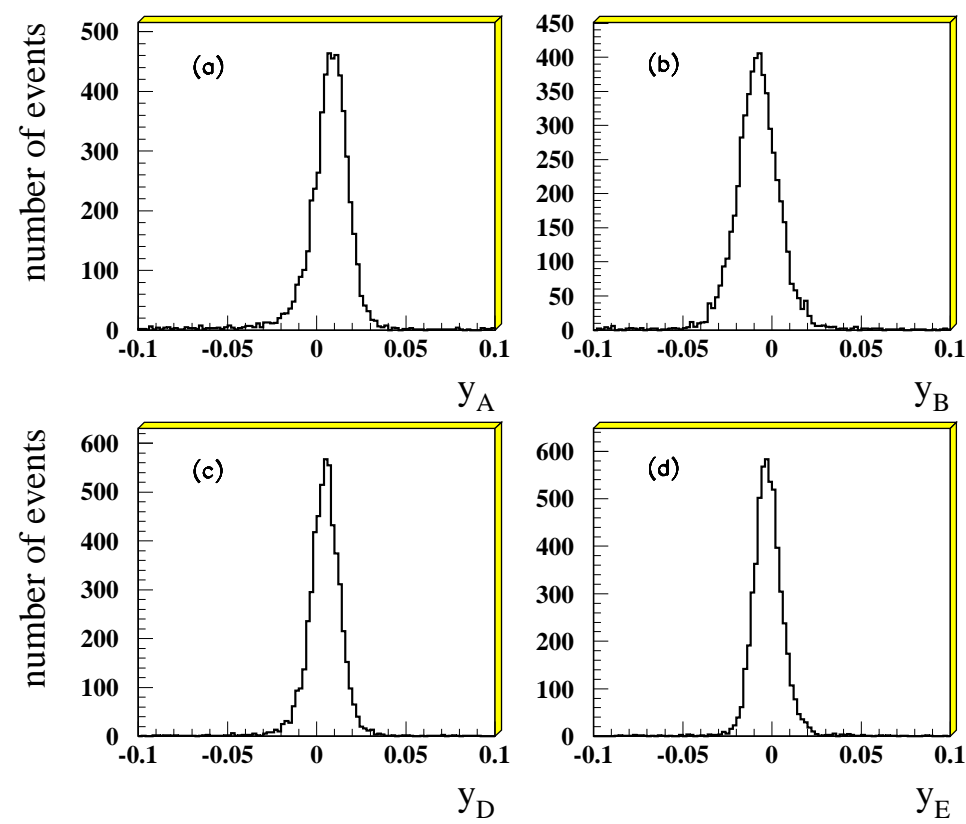

Figure 12: Distribution of the $y_{j}$ function for electrons at $40 \mathrm{GeV}$ for (a) scintillator A, (b) scintillator $B,(c)$ scintillator D and (d) scintillator E.

The dependence of scintillator response with energy using the 2 runs taken at 20 and $40 \mathrm{GeV}$ has also been studied. The energy asymmetry average values are shown in Table 6 .

Some changes with energies can be noted, for which there is no obvious reason. For each scintillator, one can compare the response at 20 and $40 \mathrm{GeV}$ (Table 7). Examples are shown in Fig. 13 for scintillator $\mathrm{C}$ and $\mathrm{F}$. The ratio of the two responses is $\sim 1.6$. 

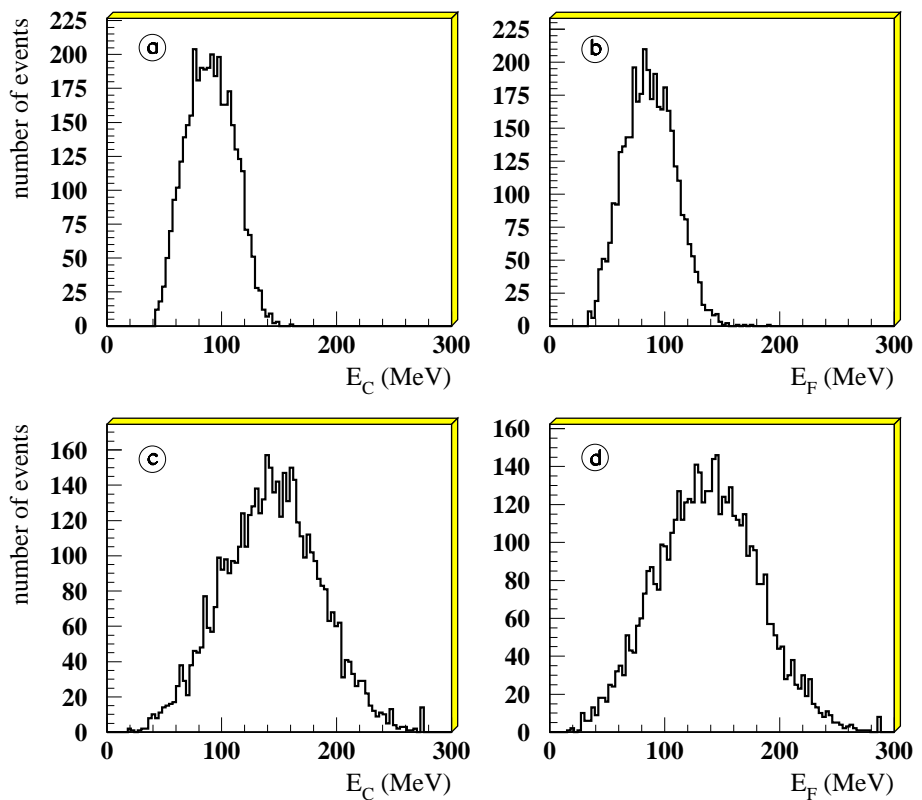

Figure 13: Distributions of energies deposited by electrons of (a) $20 \mathrm{GeV}$ in scintillator C, (b) $20 \mathrm{GeV}$ in scintillator F, (c) $40 \mathrm{GeV}$ in scintillator $\mathrm{C}$ and (d) $40 \mathrm{GeV}$ in scintillator F.

\begin{tabular}{|c|r|r|}
\hline \hline Scintillator & $20 \mathrm{GeV}$ & $40 \mathrm{GeV}$ \\
\hline \hline $\mathrm{A}$ & -0.09 & 0.60 \\
$\mathrm{~B}$ & -0.96 & -0.80 \\
$\mathrm{D}$ & 0.07 & 0.41 \\
$\mathrm{E}$ & -0.06 & 0.21 \\
\hline
\end{tabular}

Table 6: Asymmetry in the measured energies (in \%) at 20 and $40 \mathrm{GeV}$ for the 4 scintillators. 


\begin{tabular}{|c|r|r|c|}
\hline \hline Scintillator & $20 \mathrm{GeV}$ & $40 \mathrm{GeV}$ & ratio $40 / 20$ \\
\hline \hline $\mathrm{A}$ & $65(15)$ & $107(31)$ & 1.65 \\
$\mathrm{~B}$ & $66(15)$ & $108(31)$ & 1.63 \\
$\mathrm{C}$ & $90(20)$ & $143(41)$ & 1.59 \\
$\mathrm{D}$ & $78(18)$ & $124(37)$ & 1.59 \\
$\mathrm{E}$ & $101(23)$ & $159(47)$ & 1.57 \\
$\mathrm{~F}$ & $87(22)$ & $137(44)$ & 1.57 \\
\hline \hline
\end{tabular}

Table 7: The responses of all the scintillators (in $\mathrm{MeV}$ ), for 20 and $40 \mathrm{GeV}$ (RMS values in parenthesis).

\section{Summary}

Different semicircular scintillator elements with silicon photodiode readout have been tested using muon and electron beams. The results fully support the proposition to use these elements to obtain $\theta$ angle information in the upgraded L3 forward tagger at LEP II. Light yield 1000 electrons/MeV or higher have been observed, giving a signal over noise ratio of $\sim 3$ for minimum ionizing particles. The variation of light yield with particle impact point is in the range 13 to $26 \%$ over the length of the scintillators. The calibration procedure of the elements will have to take care of such non uniformities.

\section{Acknowledgments}

We acknowledge the support of the technical group of the University Claude Bernard, Lyon, and the help of the RD18 Collaboration in the setup of the data acquisition. We thank John Field for critical reading of the manuscript.

\section{References}

[1] B. Adeva et al., L3 Collaboration. Nucl. Inst. Meth. A 289 (1990) 35.

[2] B. Mouëllic Note CERN/PS202 (1989) and references therein.

[3] M. Chemarin et al., to be published.

[4] B. Bertucci et al., "Upgrade of the Active Lead Ring (ALR) for LEP200 Physics", L3 Note 1504, CERN, Geneva (Switzerland), 30 September 1993.

[5] NE102 from NES Technology S.A. Case Postale 1919, 1211 Genève 1 (Switzerland).

[6] BC408 from Bicron Corporation P.O. Box 3093, 3760 DB Soest, The Netherlands. 
[7] HAMAMATSU S6262-02 (1.5 $\mathrm{cm}^{2}$ active area) and $\mathrm{S} 3590-03\left(1 \mathrm{~cm}^{2}\right)$ from Hamamatsu Photonics, 1126-1 Hamamatsu City, 435 Japan.

[8] Millipore, Filter paper $0.45 \mu \mathrm{m}$, HAWP00010, from Millipore S.A. Saint Quentin (France).

[9] M. Goyot et al., Nucl. Inst. Meth. A 263 (1988) 180.

[10] IPN Lyon Group, "A low noise shaping amplifier with triggering channel for photodiode readout", Preprint LYCEN 8679, Université Claude Bernard, IN2P3-CNRS Lyon, and L3 Note 408, CERN, Geneva (Switzerland), 1986, modified version.

[11] ELTEC - Eurocom-7 VME system. 